\title{
Biomolecule Response to Nonthermal Plasma
}

\author{
Joshua B. Smith, Isaac Adams, \& Hai-Feng Ji \\ Department of Chemistry, Drexel University, Philadelphia, Pennsylvania, USA \\ *Address all correspondence to: Hai-Feng Ji, Department of Chemistry, Drexel University, Philadelphia, PA 19104; Tel.: \\ 01-215-895-2562, E-mail: hj56@drexel.edu
}

\begin{abstract}
This work demonstrated the decomposition products of several amino acids when exposed to nonthermal atmospheric pressure plasma. A floating electrode dielectric barrier discharge plasma was used to treat $20 \mathrm{mM}$ solutions of cysteine, glutamine, alanine, and lysine for varying treatment times to investigate possible mechanisms in cell wall degradation and treatment of tissue. The treated solutions were analyzed primarily using gas chromatography-mass spectrometry (GC-MS) and nuclear magnetic resonance (NMR). The primary targets of the plasma treatment were found to be the sulfur or nitrogen species. Acetic acid and lactic acid were observed throughout all solutions as oxidative products from the plasma treatment. This work suggests that the primary targets for plasma treatment of cell wall components is the amino acids.
\end{abstract}

KEY WORDS: amino acids, cysteine, glutamine, alanine, lysine

\section{INTRODUCTION}

Recently, thermal and nonthermal plasmas have been shown to have useful applications in agriculture. ${ }^{1}$ For example, it is generally recognized that plasmas affect plant growth, root stimulation, and microbial activation. Understanding the chemicals species generated from plasmas is required to fully develop their agricultural applications. Since cell medium consists of amino acids, glucose, vitamins, growth factors, and inorganic salts, it is important to understand what plasma treatment does to these molecules. ${ }^{2}$

This peptidoglycan layer is composed of amino acids and sugars in a polymeric network. ${ }^{4,5}$ Interference with the peptidoglycan layer can kill bacteria, as in the case with penicillin. ${ }^{4}$ It is assumed that the breakdown of biological molecules, such as amino acids, result in organic components that cause lipid peroxidation. ${ }^{2}$ Amino acid peroxides are one such species capable of causing lipid peroxidation when introduced to a lipid bilayer. The formation of amino acid peroxides as a result of reaction with hydroxyl radical has been shown by Gebicki et al. ${ }^{2}$ These studies showed that different amino acids reacted rapidly to form peroxides, whereas others had no significant effects. ${ }^{2} \mathrm{Re}-$ activity of these amino acids to form peroxides were categorized based on the number of peroxide groups formed per hydroxyl radical generated and denoted peroxidation efficiency. ${ }^{2,3}$ Kalghatgi et al. later found that peroxidation efficiency is proportional to the amount of DNA damage to cells caused by each respective amino acid after plasma treatment. $^{3}$ 
In addition to their agricultural applications, dielectric barrier discharge (DBD) plasma treatment of biomolecules is of interest for its enhanced antimicrobial effects of plasma-treated chemical solutions when compared to that of the water, ${ }^{1}$ dose-dependent effects of cell proliferation to apoptosis, ${ }^{2}$ and clinical applications where floating electrode DBD (FE-DBD) treatments are implemented. ${ }^{4}$

It is likely that reactive oxygen species (ROS) and reactive nitrogen species (RNS) generated in plasma-treated solutions oxidize and break down cell wall components. ${ }^{6-10}$ It is essential to understand the mechanisms of these reactions to explain and predict what may happen on the chemical level. Understanding the mechanism of action can better explain these effects and target additional systems where plasma treatments may prove to be useful. Some work towards a better understanding of this area has already been carried out in our laboratory through investigating the decomposition of L-valine from FE-DBD plasma treatment. ${ }^{11}$ These products included acetone, formic acid, acetic acid, threo-methylaspartic acid, erythro-methylaspartic acid, and pyruvic acid. ${ }^{11}$ The formation of threo-methylaspartic acid and erythro-methylaspartic acid verified that peroxidation of valine had occurred. ${ }^{11}$ These studies showed that the primary modes of reactions with the plasma-treated solutions are through oxidative processes. ${ }^{11}$

\section{MATERIALS AND METHODS}

Plasma treatment of biological molecules was carried out using the method and setup identical to that used by Li et al. ${ }^{11}$ L-Cysteine was purchased from Fisher Scientific. Lglutamine and caffeine were purchased from Alfa Aesar. L-Alanine, L-lysine, and deuterium oxide were purchased from Sigma Aldrich. Fresh stock solutions at a concentration of $20 \mathrm{mM}$ were made with $1 \mathrm{~mL}$ aliquots pulled for each sample treatment. Samples to be run with ${ }^{1} \mathrm{H}-\mathrm{NMR}$ analysis were prepared with $\mathrm{D}_{2} \mathrm{O}$ prior to plasma treatment. Immediately after plasma treatment, the samples were transferred to a $5 \mathrm{~mm}$ NMR tube without dilution. For quantitative studies, each NMR tube was equipped with a sealed capillary of a $10 \mathrm{mM}$ caffeine or methanol solution in $\mathrm{D}_{2} \mathrm{O}$ to be used as an external standard. Samples prepared for gas chromatography-mass spectrometry (GC-MS) analysis were run in deionized water and immediately extracted three times in dichloromethane and evaporated at room temperature to reduce the total volume to $<0.5 \mathrm{~mL}$. These samples were further processed with a trimethylsilyl (TMS) derivatization. An equal volume $(100 \mu \mathrm{L})$ of the solution and $N, O$-bis(trimethylsilyl)trifluoroacetamide (BSTFA) were then mixed and incubated for one hour at $50^{\circ} \mathrm{C}$. The solution was then diluted to 1 $\mathrm{mL}$ with dichloromethane for analysis. ${ }^{1} \mathrm{H}-\mathrm{NMR}$ spectra were recorded on a $500 \mathrm{MHz}$ Varian Unity Inova spectrometer in $5 \mathrm{~mm}$ NMR tubes with solutions made in $\mathrm{D}_{2} \mathrm{O}$. GCMS studies were carried out with a Perkin Elmer Clarus 500 capillary-column GC-MS system in positive ion mode with electron impact. 


\section{RESULTS AND DISC USSION}

\section{A. Plasma Treatment of Cysteine}

Cysteine was selected as one of the first amino acids to study to understand why such a low peroxidation efficiency was observed. According to the studies carried out by Kalghatgi et al., cysteine was found to have an extremely low peroxidation efficiency, in contrast to that of valine. ${ }^{2}$ Varying treatment times of $20 \mathrm{mM}$ L-cysteine solutions in $\mathrm{D}_{2} \mathrm{O}$ were monitored with ${ }^{1} \mathrm{H}-\mathrm{NMR}$ (Fig. 1). Using $\mathrm{D}_{2} \mathrm{O}$ as the solvent allowed for immediate analysis and prevented loss from transfer to another solvent. Cysteine itself provides two regions of peaks: a multiplet at $3.00-3.15 \mathrm{ppm}$ and a doublet of doublets at $3.95-4.00 \mathrm{ppm}$. Several additional peaks are observable in the $0-\mathrm{sec}$ solution. The signal observable at $3.35 \mathrm{ppm}$ is from an external standard, methanol in this experiment, which was added for quantitative methods. The other weak signals can be attributed to the initially oxidized form of cysteine that involves disulfide bridge formation and is otherwise known as cystine. A downfield shift of the adjacent protons is observed as these protons become further deshielded from disulfide formation. ${ }^{12}$ Plasma treatment of these solutions also causes an observable downfield shift as the $\mathrm{pH}$ of the solution decreases, which causes further protonation of the carboxylic acid group. This is most observable on the $\alpha-\mathrm{H}$ signal when a shift from $4.00 \mathrm{ppm}$ to $4.13 \mathrm{ppm}$ is observed following 3 min of plasma treatment and a decrease in $\mathrm{pH}$ from neutral down to approximately 2.2. As plasma treatment time increases, a significant decrease in the cysteine signal is observable. This is followed by an increase in the cystine concentration in the

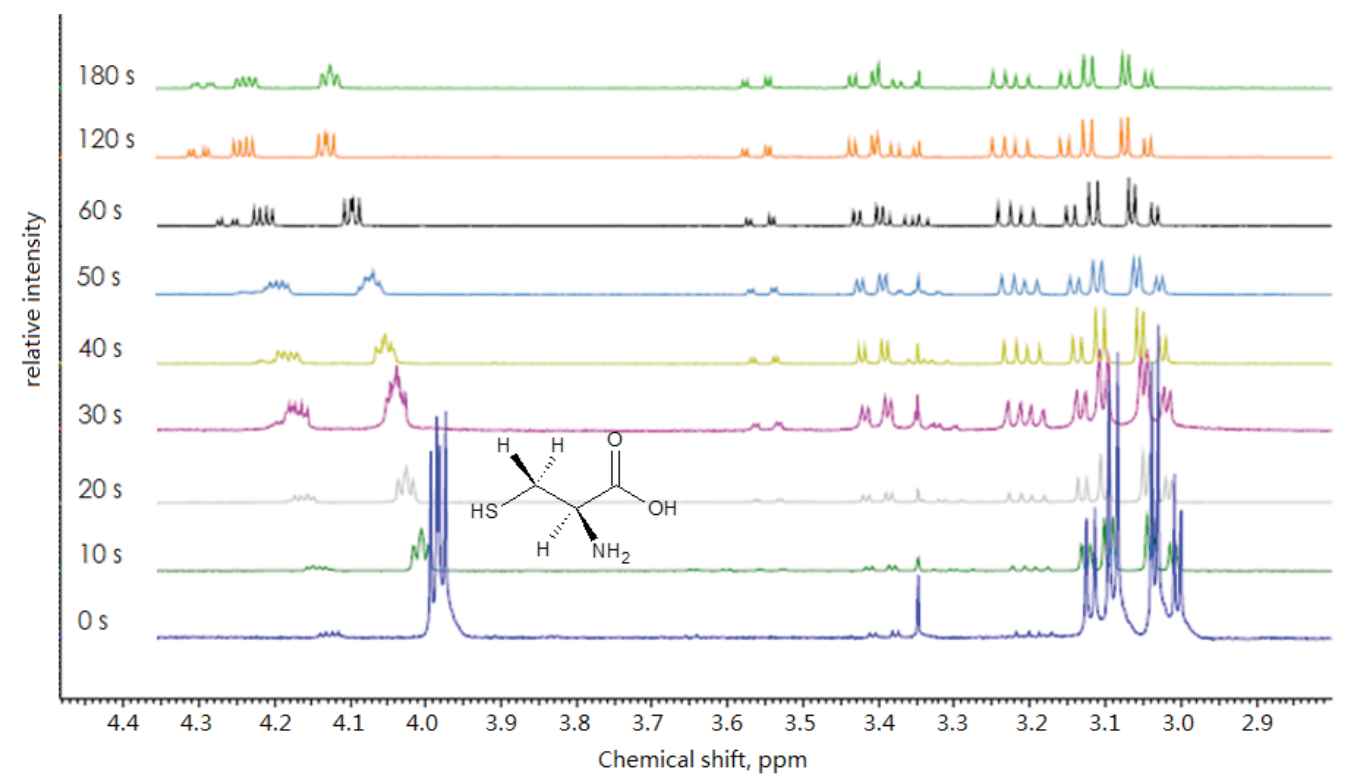

FIG. 1: Compiled ${ }^{1} \mathrm{H}-\mathrm{NMR}$ of increasing plasma treatment times on $20 \mathrm{mM}$ L-cysteine solutions

Volume 7, Issue 4, 2017 
first 30-sec treatment, until a new product is most easily observed after 30-sec treatment times. This next set of peaks has been attributed to cysteic acid formation, which is the expected oxidation product under acidic conditions. ${ }^{12} 2 \mathrm{D}$-correlation spectroscopy (2DCOSY) experiments verified the coupling of these three pairs of signals by matching the adjacent protons (Fig. 2). Plasma treatment times were extended to 5, 10, and $20 \mathrm{~min}$, and the only newly formed peaks belonged to that of formic acid $(8.20 \mathrm{ppm})$ and acetone $(2.06 \mathrm{ppm})$. It should be noted that these were present in very low concentrations and were only first observable in minute amounts after 5 min of plasma treatment.

This suggests a much different story to what was observed with valine. Plasma treatment of cysteine was found to follow the similar path of standard oxidation (Fig. 3). ${ }^{12,13}$

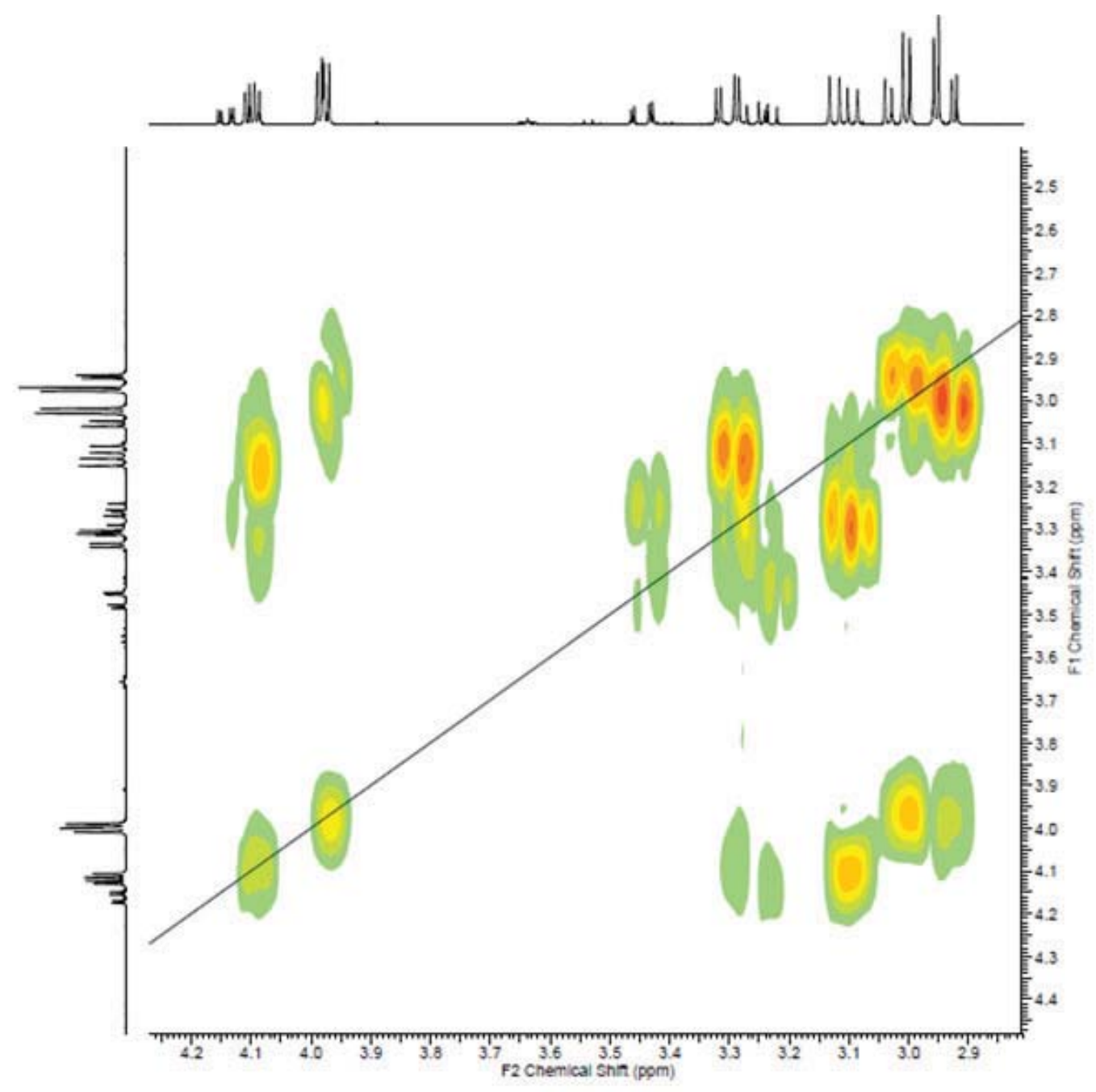

FIG. 2: 2D-COSY on 1-min plasma-treated $20 \mathrm{mM}$ L-cysteine 


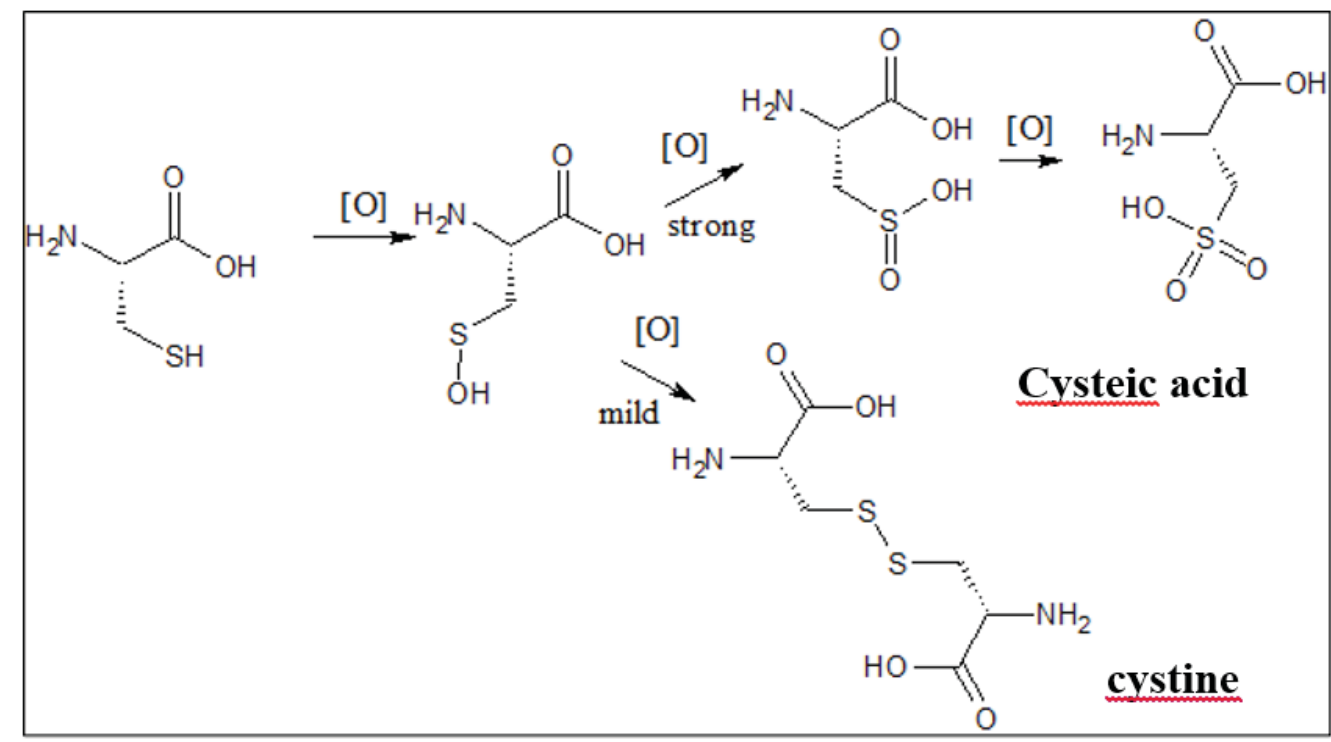

FIG. 3: Oxidation pathway of L-cysteine to cystine and cysteic acid

These results were also validated with GC-MS studies (see Fig. A2 in Appendix A). This work explains that the low peroxidation efficiency of plasma-treated cysteine can be attributed to the strong antioxidant role of the thiol functionality. ${ }^{14}$ The antioxidant capabilities of the thiol protect the molecule from further degradation. This logic can also be used to explain the lower peroxidation efficiency observed with methionine.

\section{B. Plasma Treatment of Glutamine}

For glutamine, similar studies on the effects of plasma treatment were monitored with ${ }^{1} \mathrm{H}-\mathrm{NMR}$ (Fig. 4). The glutamine concentration noticeably decreased following the 3-min plasma treatment; however, detection of additional products was difficult. Many oxidation products of glutamine result in overlapping ${ }^{1} \mathrm{H}-\mathrm{NMR}$ signals, specifically glutamic acid, as these two compounds are very similar in structure. Some information could still be derived from the NMR data. Fumaric acid was not detected from the degradation, as no peak was present at $6.5 \mathrm{ppm}$ in the proton NMR. ${ }^{15}$ The formation of $\alpha$-ketoglutaric acid was observed from the NMR with a multiplet forming around 3 ppm at $50 \mathrm{sec}$ of plasma treatment. ${ }^{15}$ No other compounds were discernable from the ${ }^{1} \mathrm{H}-\mathrm{NMR}$ data because many of the probable compounds result in overlapping signals within the same region.

GC-MS studies were similarly carried out with an identical method to that of cysteine for the plasma treated glutamine. Derivatization with BSTFA resulted in several peaks from residual components and solvent (Fig. A4 in Appendix A). These peaks were found at $1.61,1.63,1.73,1.91$, and $3.55 \mathrm{~min}$ and are attributed to the dichloromethane solvent 


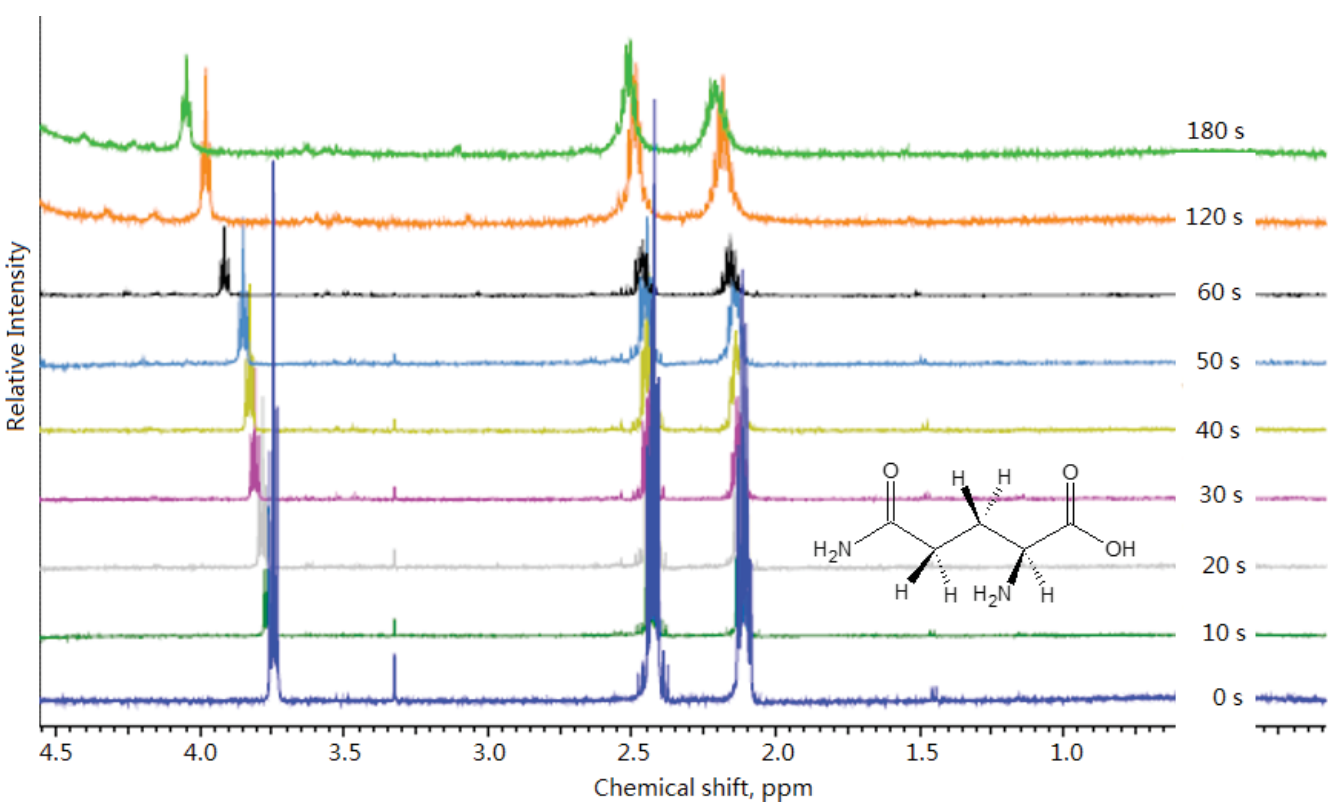

FIG. 4: Compiled ${ }^{1} \mathrm{H}-\mathrm{NMR}$ of increasing plasma treatment times on $20 \mathrm{mM}$ L-glutamine solutions

and residual components from trimethylsilyl-derivatization. ${ }^{11}$ The chromatograms were found to have a predominant peak at $6.96 \mathrm{~min}$, which was found to belong to the coelution of glutamic acid and 2-hydroxypentanedioic acid. It is likely that oxalic acid and pyruvic acid were also formed; however, these were not confirmed with GC-MS, and if present, their concentration was probably very low. Acetic acid was recognized at $2.18 \mathrm{~min}$ and was determined from the representative peak at $117 \mathrm{~m} / \mathrm{z} \cdot{ }^{16}$ Lactic acid was found at a retention time of $2.34 \mathrm{~min}$. The presence of acetic acid and lactic acid shows the susceptibility of the carbon chain to fragmentation from plasma treatment in solution. No additional major products were detectable from glutamine, but the suggested pathway of oxidation is shown in Fig. 5. The treatment of glutamine shows not only oxidation but cleavage of the hydrocarbon chain as a possible pathway.

\section{Plasma Treatment of Additional Biomolec ules}

Investigations into additional amino acids included alanine and lysine. GC-MS studies on the plasma treatment of alanine showed the formation of acetaldehyde (2.689 $\mathrm{min}$ ) as the major product, along with lactic acid (2.354 min). The plasma treatment of lysine similarly resulted in the formation of acetic acid. Lactic acid formation was also observed with an identical retention time to what was previously observed. The primary component was 2-amino-6-hydroxyhexanoic acid, which is formed from the loss of the terminal amine group followed by the addition of hydroxide to that carbon. This was observed at a retention time of 7.026 min. Further investigation of the fragmentation 


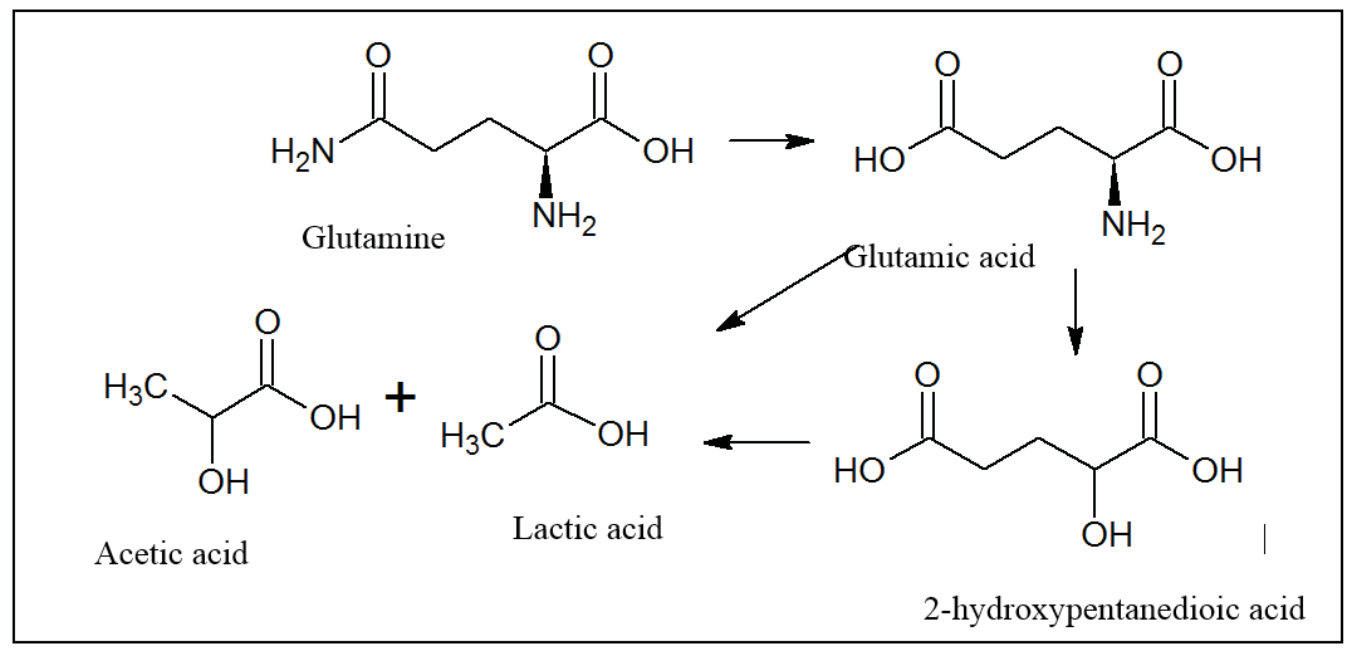

FIG. 5: Oxidation pathway observed for glutamine with plasma treatment

revealed that another compound co-eluted at this time. It is likely that 2,6-dihydroxyhexanoic acid also formed and would have a very similar retention time. This GC-MS data with chromatograms are included in Appendix A. The treatment of the amino acid solutions has revealed primarily oxidation products with a common scheme of amine abstraction followed by a replacement with an alcohol functionality. The main groups that were susceptible to the reactive plasma components were those most likely to be oxidized, including the sulfur and nitrogen functionalities. Additionally, the hydrocarbon chain was susceptible to cleavage. This suggests that hydroxyl radicals are present in significant concentration and this could be explained by the large amount of hydrogen peroxide generated in solution. The difficulty with concluding that only hydrogen peroxide was responsible for these components comes from the fact that no peroxide species were isolated during these experiments. This suggests that additional species could be responsible for the oxidation and assist in the degradation of these species in solution. Additional conclusions can be drawn from these studies to explain the effects of plasma treatment on the bacterial cell wall. The cell wall is comprised of a peptidoglycan layer, as mentioned earlier in this section. This peptidoglycan layer is linked together by peptide linkages between the amino acids and glycosidic linkages between the sugar backbone. The rapid breakdown of these amino acids suggests that the amino acid bridges may be the first target of plasma treatment of the peptidoglycan layer. For a comparison, brief studies were carried out with sucrose.

Sucrose is an example of a basic glycosidic linkage between glucose and fructose. Plasma treatment of sucrose was first investigated using Benedict's solution. Benedict's solution changes color from blue to red upon reduction of $\mathrm{Cu}^{2+}$ to $\mathrm{Cu}^{1+}$. Benedict's reagent was tested on sucrose solutions treated with plasma for $30 \mathrm{sec}, 1 \mathrm{~min}$, and $5 \mathrm{~min}$; a trend towards increasing change of blue to red was observed (Fig. 6). This was matched 
against control solutions of sucrose, glucose, and fructose, in which colors of blue, red, and red were observed, respectively. This shows that upon the presence of a reducing sugar such as glucose, the reduction of copper occurs. One of the issues with this test is that there can be many false positives. Also, from the previous water studies, it is known that a large amount of $\mathrm{H}_{2} \mathrm{O}_{2}$ is formed in solution, and hydrogen peroxide has been known to serve as both a reducing and oxidizing agent. ${ }^{17}$

To further understand how the sucrose molecules are breaking down, it is necessary to study this system with a different analysis. ${ }^{1} \mathrm{H}-\mathrm{NMR}$ studies were investigated on 20 $\mathrm{mM}$ solutions of sucrose treated with plasma for $5 \mathrm{~min}$ (Fig. 7). Following $5 \mathrm{~min}$ of plasma treatment, a large amount of sucrose was found to still be present. However, a number of new compounds were observable with NMR. The first and most obvious was the formation of formic acid, as recognized from the signal at $8.2 \mathrm{ppm}$. Also, looking at the inset from Fig. 7, the formation of glucose was apparent from the signals at 4.55 ppm. Some additional species that could be detected included glyceric acid (4.3 ppm), tartronic acid $(5.3 \mathrm{ppm})$, and tartaric acid $(4.65 \mathrm{ppm})$. These compounds were further characterized and analyzed with GC-MS studies, and the mechanism of formation was discussed by Li et al. ${ }^{18}$

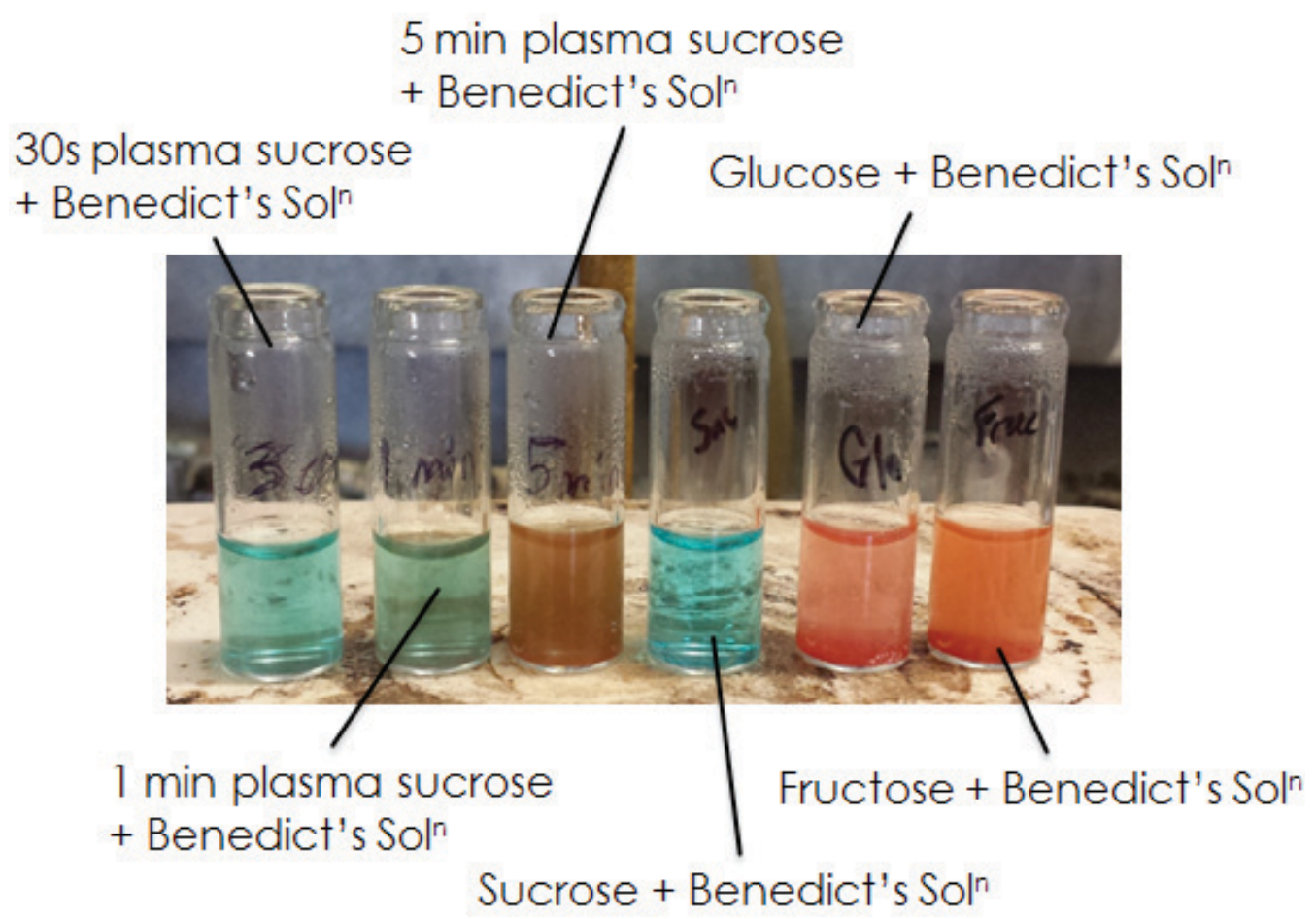

FIG. 6: Benedict's test with plasma treated sucrose after $30 \mathrm{sec}, 1 \mathrm{~min}$, and $5 \mathrm{~min}$ (left 3). The control test with sucrose maintains the blue color and the solution changes to red on exposure to glucose and fructose (right 3 ). 


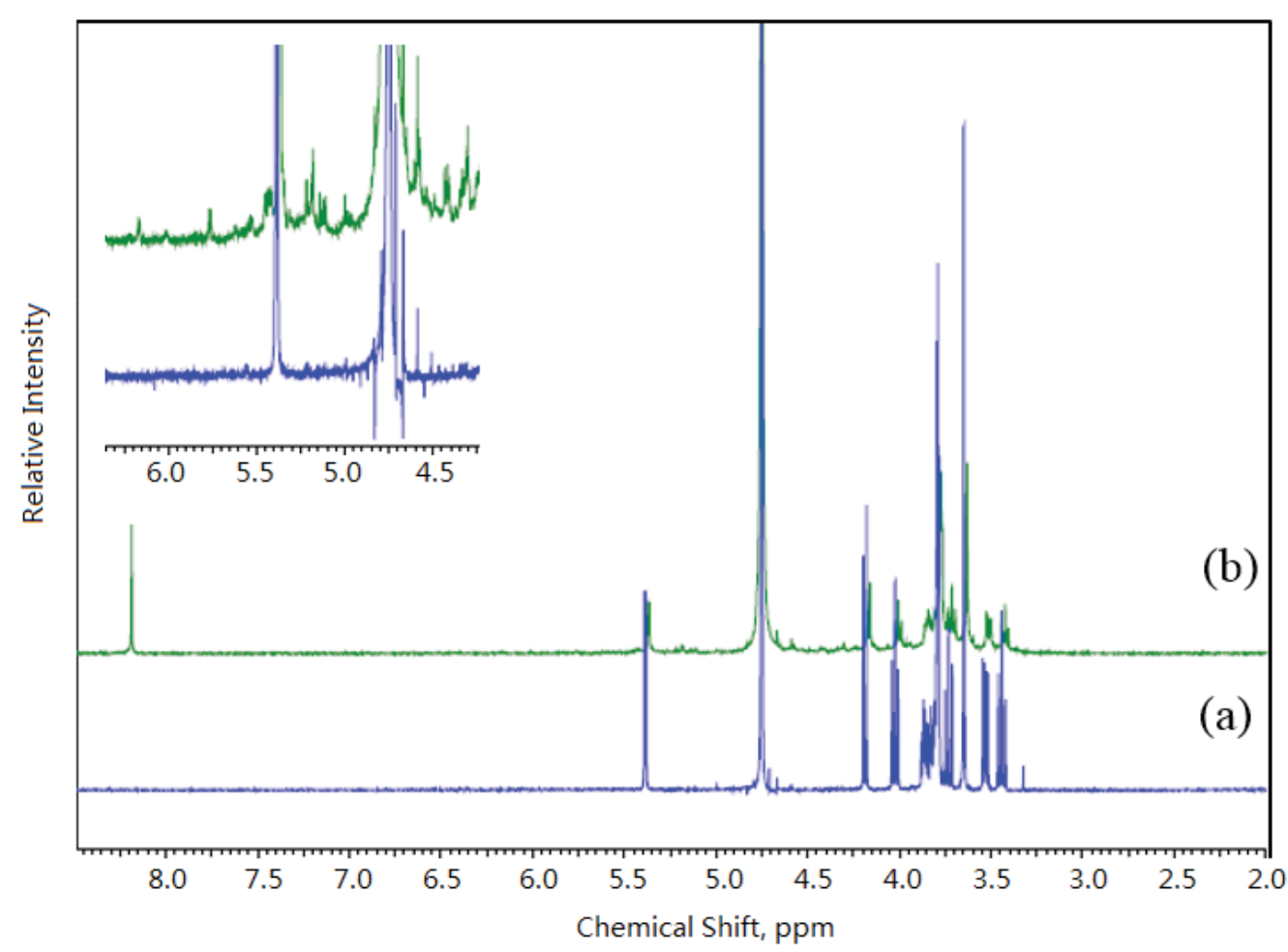

FIG. 7: ${ }^{1} \mathrm{H}-\mathrm{NMR}$ of $20 \mathrm{mM}$ sucrose solutions at (a) $0 \mathrm{~min}$, and (b) after $5 \mathrm{~min}$ of plasma treatment

\section{CONCLSION}

Plasma treatment of amino acids has led to a deeper understanding into some of the effects on solutions. The amino acid functionalities play a significant role on the effects of plasma treatment. The primary targets are any sulfur or nitrogen functionalities. The primary reactions with the sulfur functionalities was simply oxidation, however, nitrosylation was observed during the characterization of N-acetyl cysteine (NAC) solutions. ${ }^{19}$ No oxidation of the amine functionality was observed, but it is likely that it occurred and was rapidly followed by removal and replacement with a hydroxyl functionality. It is likely that the amine groups contributed to the nitric acid concentration in solution; however, further studies would need to be carried out to completely verify this. An increase of plasma treatment times yielded fragmentation along the carbon backbone. This was most likely caused by the highly oxidizing and acidic conditions. The presence of hydroxyl radicals allows for hydrogen abstraction and opens up the carbon for further reactions. The peroxidation efficiency of these amino acids can be explained by the differences in the functionalities. The primary reasoning for the sulfur-containing amino acids having such low peroxidation efficiency can be explained by the antioxidant capabilities. Sulfur is much more easily oxidized than the other elements present.

Volume 7, Issue 4, 2017 
Glutamine was found to have a peroxidation efficiency towards the middle of the group. This can likely be explained by the presence of two amine groups, which doubles the reactive sites for oxidation and likely decreases cleavage of the $\mathrm{C}-\mathrm{C}$ bond. This was demonstrated by the increased amount of glutamic acid and 2-hydroxypentanedioic acid in the GC-MS studies. Also, acetic acid and lactic acid were found, which suggests that longer plasma treatment times caused the $\mathrm{C}-\mathrm{C}$ bond to break down. Glutamic acid likely has a higher peroxidation efficiency because only one amine group is present, so more energy goes into the $\mathrm{C}-\mathrm{C}$ bond cleavage. Lysine was found to break down into 2-amino-6-hydroxyhexanoic acid, and this was further oxidized to 2,6-dihydroxyhexanoic acid. Both of these compounds have a longer carbon chain, which provides more reactive sites for fragmentation and breakdown. It is likely that this occurred as well, because lactic acid and acetic acid were found in even higher concentrations than in the glutamine solutions.

The degradation of sucrose showed that plasma treatment can be used for breakdown of the glycosidic linkage. Presence pf glucose was verified in the NMR studies; however, it took a much longer plasma treatment time to observe the result of any reaction with plasma. This suggests that breakdown of the glycosidic linkage could possibly explain the plasma reactivity to the bacterial cell wall, but it is more likely to occur on the amino acid network. This does show a potential application for breakdown of cellulose to glucose with plasma treatment for potential applications in the energy and even possibly food fields.

\section{REFERENCES}

1. Ercan UK, Wang H, Haifang J, Fridman G, Brooks AD, Joshi SG. Nonequilibrium plasma-activated antimicrobial solutions are broad-spectrum and retain their efficacies for extended period of time. Plasma Process Polym. 2013;10:544-55.

2. Kalghatgi S, Kelly CM, Cerchar E, Torabi B, Alekseev O, Friedman G, Azizkhan-Clifford J. Effects of non-thermal plasma on mammalian cells. PLoS One. 2011;6:1-11.

3. Gebicki S, Gebicki JM. Formation of peroxides in amino acids and proteins exposed to oxygen free radicals. Biochem J. 1993;289(3):743-9.

4. Lodish H, Berk A, Zipursky S, Matsudaira P, Baltimore D, Darnell J. Molecular cell biology. 4th ed. New York: Freeman; 2000.

5. Vollmer W, Blanot D, De Pedro MA. Peptidoglycan structure and architecture. FEMS Microbiol Rev. 2008;32(2):149-67.

6. Yusupov M, Bogaerts A, Huygh S, Snoeckx R, van Duin ACT, Neyts EC. Plasma-induced destruction of bacteria cell wall components: a reactive molecular dynamic simulation. J Phys Chem C. 2013;117(11):5993-8.

7. Ma Y, Zhang G, Shi X, Xu G, Yang Y. Chemical mechanisms of bacterial inactivation using dielectric barrier discharge plasma in atmospheric air. IEEE Trans Plasma Sci. 2008; 36:1615-20.

8. Şen Y, Mutlu M. Sterilization of food contacting surfaces via non-thermal plasma treatment: a model study with Escherichia coli-contaminated stainless steel and polyethylene surfaces. Food Bioproc Tech. 2013;6:3295-304.

9. Miao H, Yun G. The sterilization of Escherichia coli by dielectric-barrier discharge plasma at atmospheric pressure. Appl Surf Sci. 2001;257(16):7065-70.

10. Kostov KG, Rocha V, Koga-ito CY, Matos BM, Algatti MA, Honda RY, Kayama ME, Mota RP. Bac- 
terial sterilizationi by a dielectric barrier discharge (DBD) in air. Surf Coat Technol. 2010;204(1819):2954-9.

11. Li Y, Kojtari A, Friedman G, Brooks AD, Fridman A, Ji HF. Decomposition of L-Valine under nonthermal dielectric barrier discharge plasma. J Phys Chem B. 2014;118(6):1612-20.

12. Katritzky AR, Akhmedov NG, Denisko OV. ${ }^{1} \mathrm{H}$ and ${ }^{13} \mathrm{C}$ NMR spectroscopic study of oxidation of D,Lcystine and 3,3'-dithiobis(propionic acid) with hydrogen peroxide in aqueous solution. Magn Reson Chem. 2003;41:37-41.

13. Enescu M, Cardey B. Mechanism of cysteine oxidation by a hydroxyl radical: a theoretical study. Chemphyschem. 2006;7:912-9.

14. Kerksick C, Willoughby D. The antioxidant role of glutathione and $\mathrm{N}$-acetyl-cysteine supplements and exercise-induced oxidative stress. J Int Soc Sports Nutr. 2005;2(2):38-44.

15. Wishart DS, Knox C, Guo AC, Eisner R, Young N, Gautam B, Hau DD, Psychogios N, Dong E, Bouatra S, Mandal R, Sinelnikov I, Xia J, Jia L, Cruz JA, Lim E, Sobsey CA, Shrivastava S, Huang P, Liu P, Fang L, Peng J, Fradette R, Cheng D, Tzur D, Clements M, Lewis A, De Souza A, Zuniga A, Dawe M, Xiong Y, Clive D, Greiner R, Nazyrova A, Shaykhutdinov R, Li L, Vogel HJ, Forsythe I. HMDB: a knowledgebase for the human metabolome. Nucleic Acids Res. 2009;37(Database):D603-10.

16. Rousová J, Ondrušová K, Karlová $\mathrm{P}$, Kubátová A. Determination of impurities in bioproduced succinic acid. J Chromatogr Sep Tech. 2016;6(2):264.

17. Bancroft WD, Murphy NF. Oxidation and reduction with hydrogen peroxide. J Phys Chem. 1934;39(3):377-98.

18. Li Y, Friedman G, Fridman A, Ji HF. Decomposition of sugars under non-thermal dielectric barrier discharge plasma. Clin Plasma Med. 2014;2(2):56-63.

19. Ercan U, Smith J, Ji HF, Brooks AD, Joshi SG. Chemical changes in nonthermal plasma-treated Nacetylcysteine (NAC) solution and their contribution to bacterial inactivation. Sci Rep. 2016;6:20365.

\section{APPENDIX A: GC-MS DATA WTH CHROMATOGRAMS}

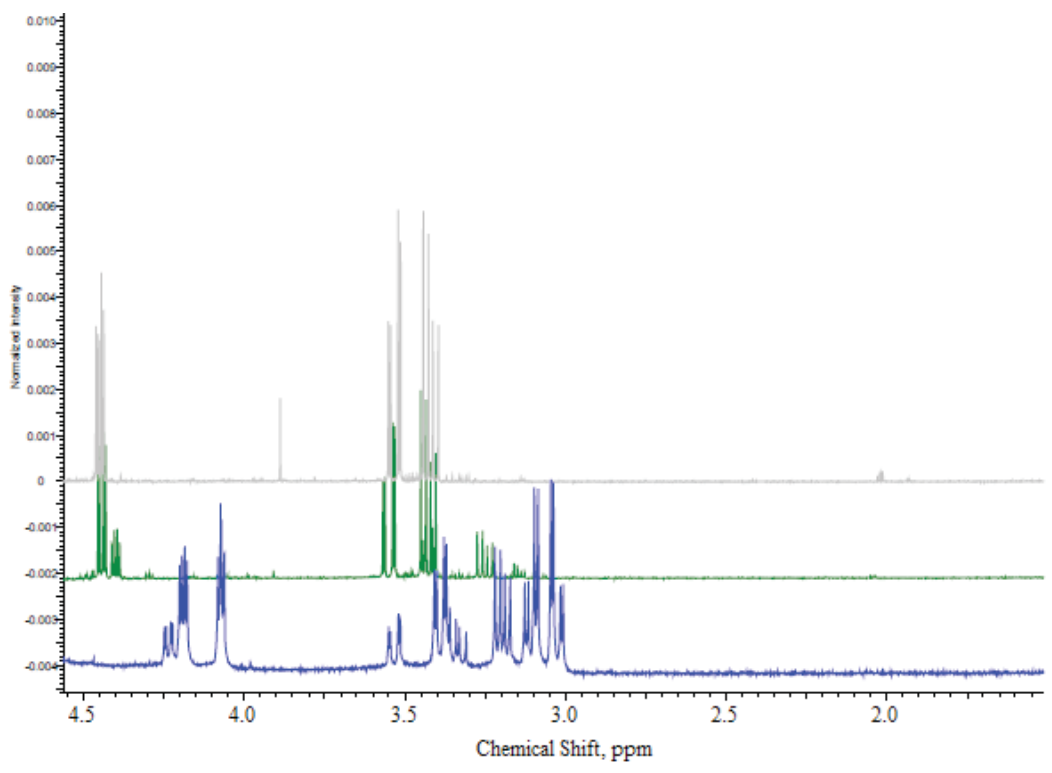

FIG. A1: ${ }^{1} \mathrm{H}-\mathrm{NMR}$ of plasma treated L-cysteine solutions for 1,5 , and 10 min treatment times showing formation of cysteic acid

Volume 7, Issue 4, 2017 


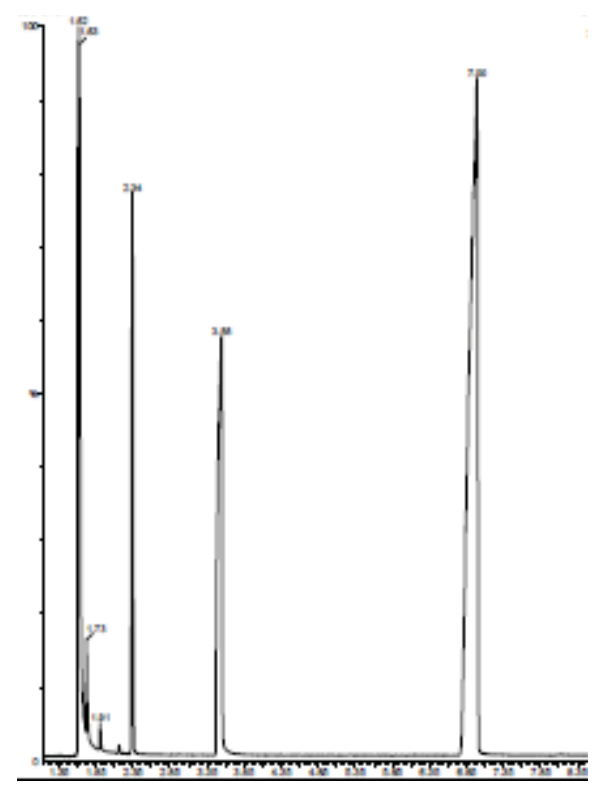

FIG. A2: GC chromatogram of 3-min plasma treated cysteine

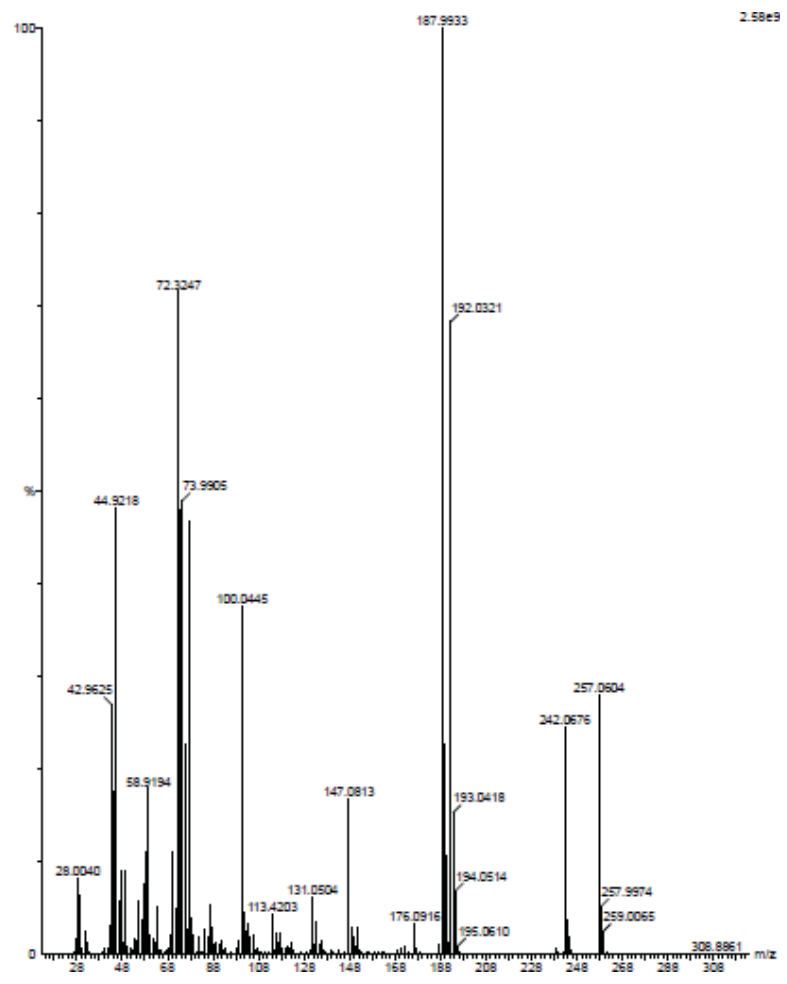

FIG. A3: MS data from GC peak at $6.986 \mathrm{~min}$ in plasma treated cysteine 


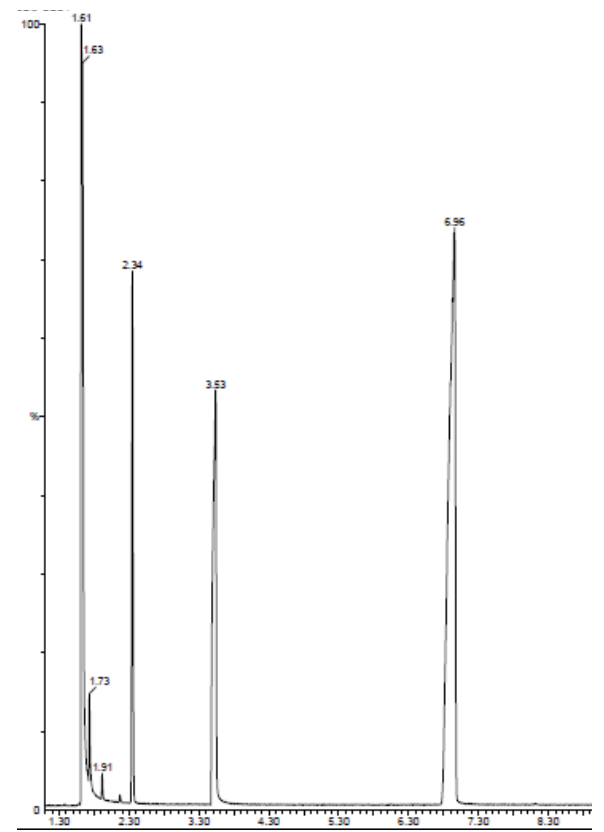

FIG. A4: GC chromatogram from 3-min plasma treated glutamine

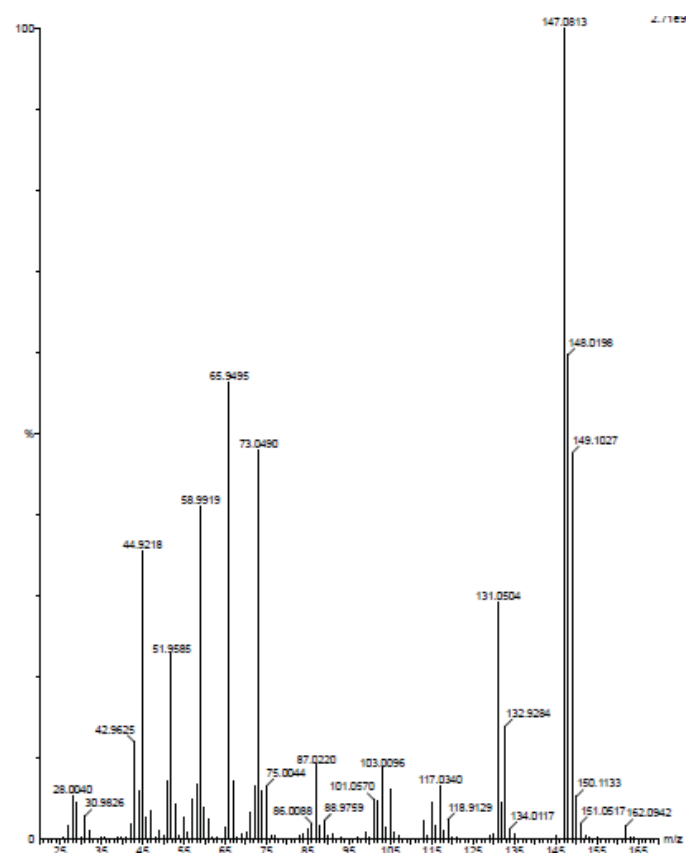

FIG. A5: MS data from GC peak at $2.349 \mathrm{~min}$ in plasma treated glutamine 


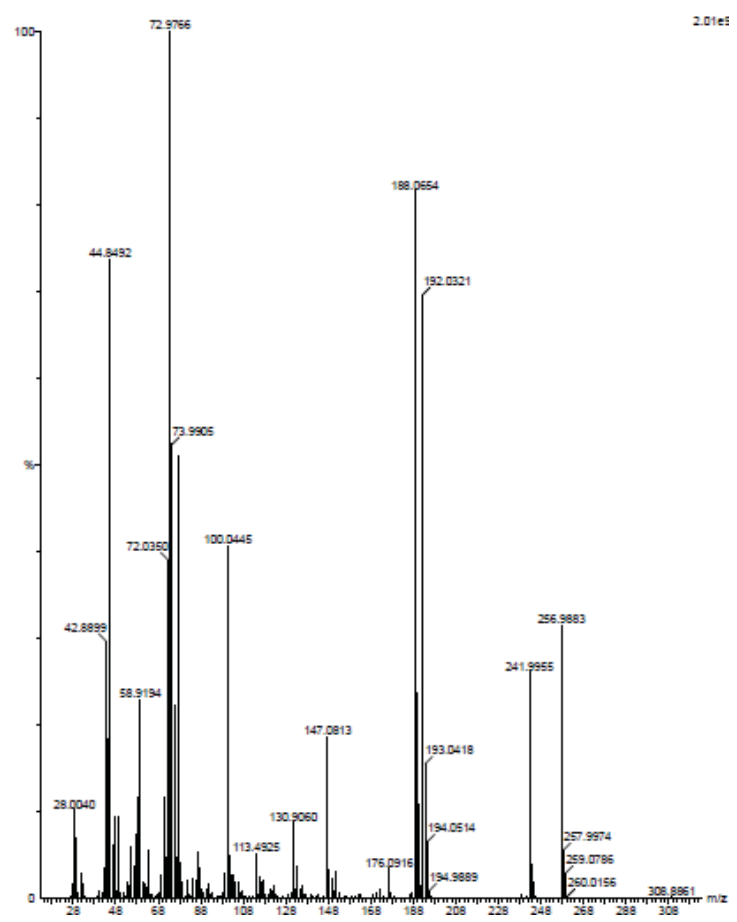

FIG. A6: MS data from GC peak at $6.961 \mathrm{~min}$ in plasma treated glutamine

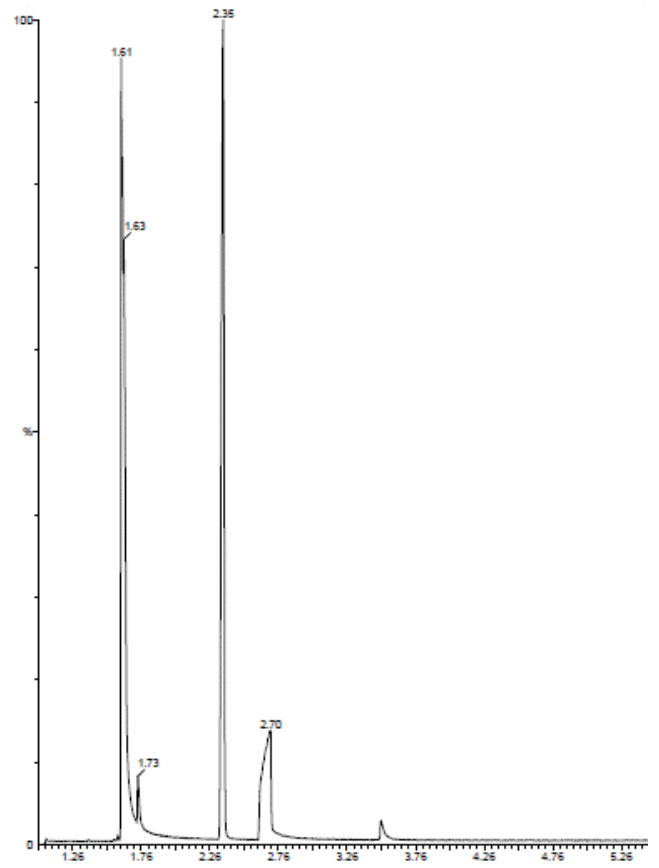

FIG. A7: GC chromatogram of 3-min plasma treated alanine 


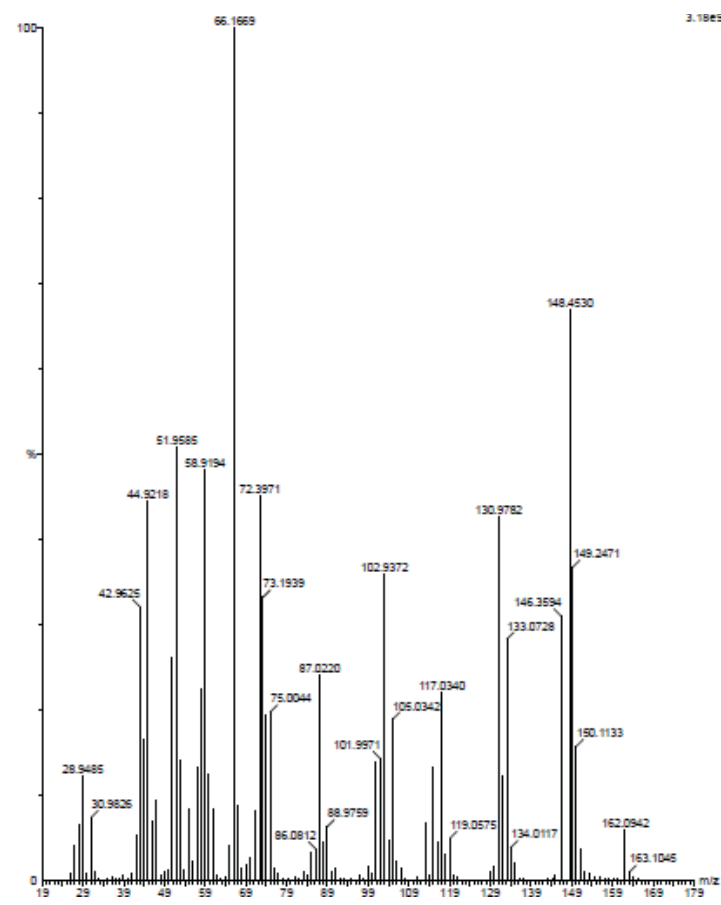

FIG. A8: MS data from GC peak at $2.354 \mathrm{~min}$ in plasma treated alanine

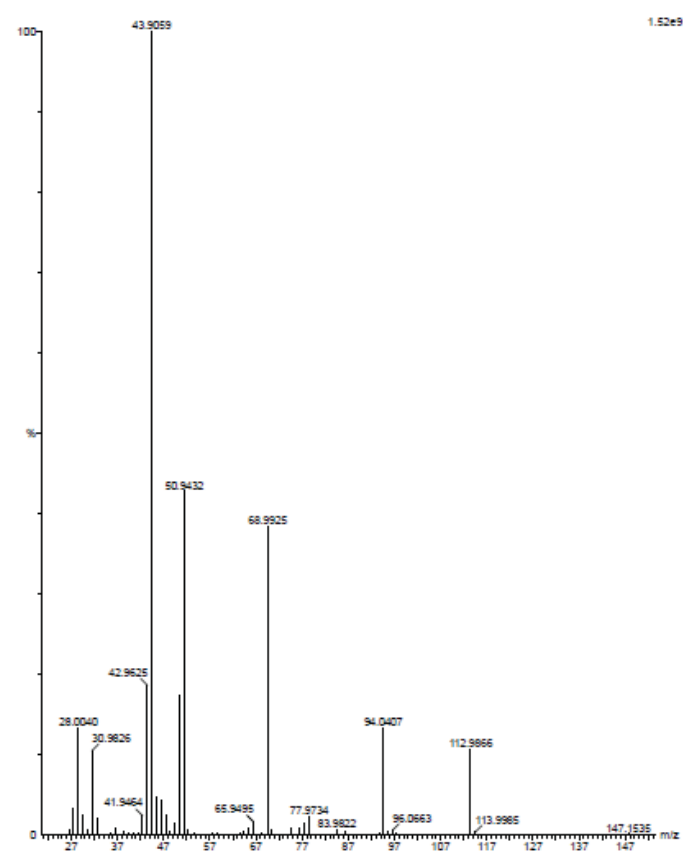

FIG. A9: MS data from 2.689 min plasma treated alanine 


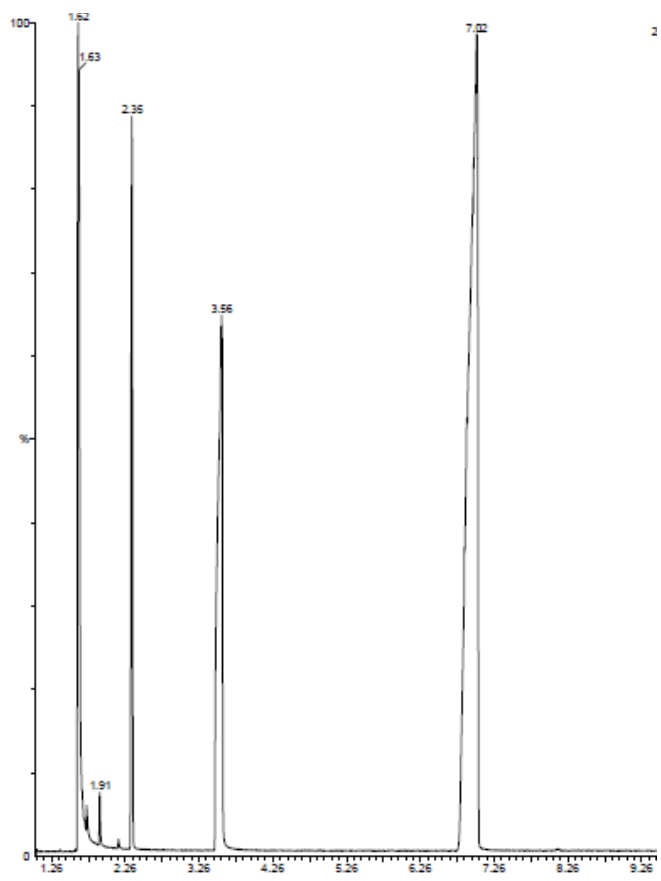

FIG. A10: GC chromatogram of 3-minute plasma treated lysine

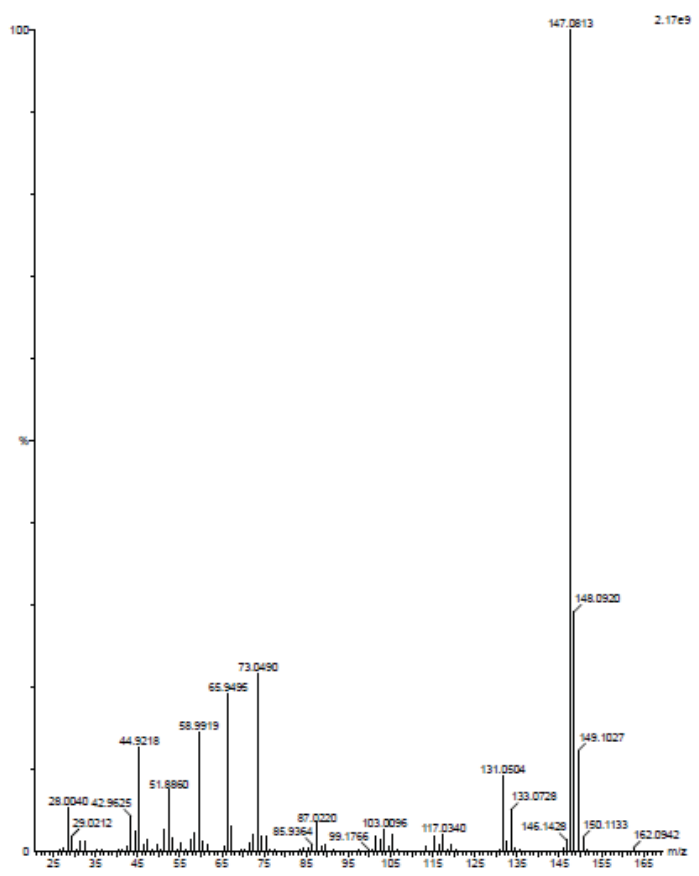

FIG. A11: MS data from $2.359 \mathrm{~min}$ plasma treated lysine 


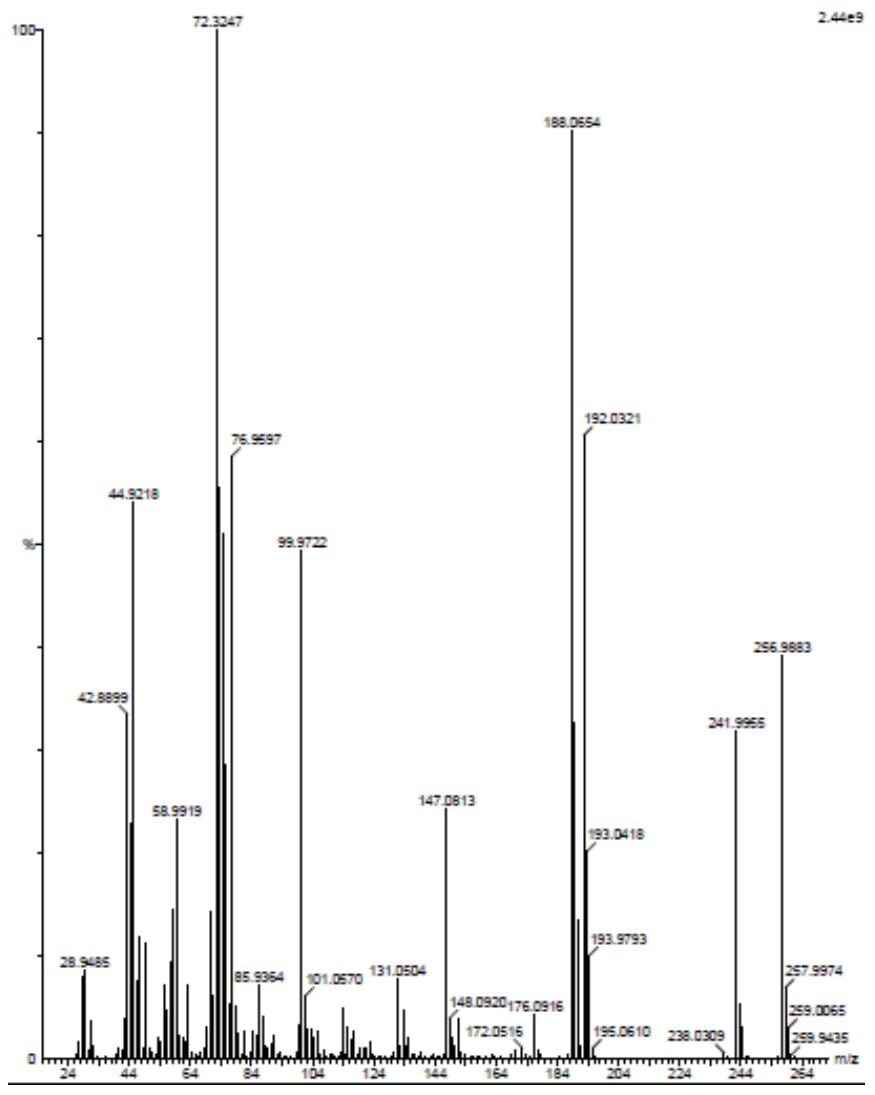

FIG. A12: MS data from GC peak at 7.026 min for plasma treated lysi 